$\begin{gathered}\text { Revista do Departamento de Geografia } \\ \text { Universidade de São Paulo } \\ \text { www.revistas.usp.br/rdg }\end{gathered}$
V.32 (2016)
ISSN 2236-2878

\title{
Indústria Cultural e Conhecimentos Tradicionais: A Associação Naboa e a Territorialização do Artesanato em Anchieta (ES)
}

\section{Cultural Industry and Traditional Knowledge: The Naboa Association and The Territorialization of Artisanal Handcraft in Anchieta (ES)}

Recebido (Received): 09/06/2016 DOI: $10.11606 /$ rdg.v2i0.116296

\author{
Rodrigo Ramos Hospodar Felippe Valverde \\ Universidade de São Paulo \\ rvalverde@usp.br
}

Resumo: O objetivo deste artigo é analisar a transformação da atividade artesanal tradicional no município de Anchieta (ES). Tal transformação é realizada pela conversão das técnicas e valores tradicionais por outros, resultado das novas trocas com atores como a SAMARCO Mineração e o SEBRAE-ES, além da ampliação e diversificação do seu mercado consumidor. Seus produtos têm recebido premiações internacionais (MERCOSUL, UNESCO), ganhando espaço no mercado globalizado, ao mesmo tempo em que capacitações e parcerias são desenvolvidas para cumprir os rigores dos consumidores. Ainda mais importante para um estudo geográfico, o novo artesanato de Anchieta que se observa na Associação NABOA parece trazer consequências territoriais, configurando os termos de uma nova indústria cultural, tal qual esta foi definida por Power e Scott. Estas consequências se realizam tanto física quanto simbolicamente, por intermédio da construção da sede para a Associação e pelo contínuo processo de incorporação de valores ao processo produtivo.

Palavras-chave: Artesanato, Indústria Cultural, Anchieta, Territorialização.

\begin{abstract}
The objective of this paper is to analyze the transformation of traditional handicraft activity in the municipality of Anchieta (ES). This transformation is accomplished through the conversion of techniques and other traditional values, due to the new exchanges with actors like SAMARCO Mining and SEBRAE-ES, as well as the expansion and diversification of its consumer market. Its products had been awarded with international prizes (UNESCO, MERCOSUR), gaining visibility in the global market, while partnerships and training courses were developed to fulfill new consumer's standards. Even more important for a geographical study, the new craft of Anchieta as observed in the NABOA Association seems to bring territorial consequences, setting the terms of a new cultural industry, as described by Power and Scott. These territorial consequences are both physical and symbolic, constituted through the construction of the Association's headquarters and the continuous incorporation of external values to the productive process.
\end{abstract}

Keywords: Craft, Cultural Industry, Anchieta, Territorialization. 


\section{INTRODUÇÃO}

A cidade de Anchieta (ES, pouco mais de 23.000 habitantes) é reconhecida pela presença econômica da mineradora SAMARCO (de propriedade da Vale S.A. e da BHP Billiton) e pela indústria do turismo, que é viabilizada pelos balneários (Lagoa Mãe-Bá, Praias de Ubu e Iriri, entre outras), pelo patrimônio histórico (Igreja Matriz Nossa Senhora Assunção) e pelos eventos culturais (carnaval, shows etc.). Do contato de atividades econômicas tão distintas, o que se destaca é o atrito entre os subprodutos da atividade mineradora (mercúrio nas águas e poluição do ar) e a necessidade da qualificação da experiência do turismo pela inviolabilidade da natureza. Em ambos os casos, observam-se dificuldades para que as comunidades residentes (Mãe-Bá, Porto Grande, Condados, Chapada do Á e Meaípe) na região ganhem qualidade de vida.

Diversas vezes autuada pelo despejo de dejetos na Lagoa Mãe-Bá e no mar desde 1993, a SAMARCO aspira alcançar nova legitimidade por intermédio de uma política de responsabilidade social e sustentabilidade. Entendem-se tais políticas por uma série de ações pontuais de financiamento de projetos relativos à recuperação do ambiente ou a alguma qualidade de vida (oficinas de reciclagem de lixo, jornadas de conscientização dos riscos de doenças etc.). Tratam-se de ações eventuais, em pequena escala geográfica e com pouco efeito de territorialização, feitas para melhorar a visibilidade da empresa. É preciso destacar que tais ações não aspiram substituir em peso ou em regularidade as políticas públicas: seus movimentos se definem pelo marketing e seus efeitos sobre o consumo. A expansão destas práticas revela uma reação às formas de regulamentação pública de suas atividades e às informações que possam influenciar os consumidores.

O uso da cultura como elemento de legitimação de empresas e os limites de territorialização em seus movimentos compõem o núcleo desse artigo. Como modo de ação empresarial, a cultura apresenta maior flexibilidade, menores custos e maior retorno em relação às ações de recuperação do meio-ambiente, devido a velocidade de seus ciclos de produção e consumo. Esta cultura não é a expressão de um saber-fazer fixo, reproduzida geração após geração em uma região, e tampouco fruto do conhecimento da natureza. Por oposição, assumimos a perspectiva de Mitchell:

Não existe tal coisa (ontológica) que chamamos de cultura. Em vez disso, há apenas uma idéia muito poderosa de cultura, uma idéia que se desenvolveu sob condições históricas específicas e posteriormente ampliou-se como um meio de explicar diferenças materiais, ordem social e relações de poder (...). Mas estas explicações não são da "cultura propriamente dita", quer definida como um nível, meio ou sistema de significações. Estas maneiras de ver a "cultura" não impedem a reificação, mas a perpetuam, introduzindo no cerne da geografia o que ainda é um conjunto mistificado de suposições sobre como se realiza a prática social. E isto permanecerá até que os teóricos da sociedade abandonem a noção de uma cultura ontológica e comecem a focalizar como a própria idéia de cultura foi desenvolvida e desdobrada como um meio de tentar ordenar, controlar e definir "outros" em nome do poder ou do lucro (MITCHELL, 2008, p.83).

É nesse contexto que, em 2005, se desenvolveu uma parceria entre a SAMARCO, as comunidades de pescadores e o SEBRAE-ES para o desenvolvimento do projeto TABOA LAGOA e para a criação do Núcleo de Artesanato em Taboa (NABOA). A SAMARCO criou o projeto com o objetivo de minimizar os conflitos locais e regionais por intermédio do financiamento e promoção de uma política de responsabilidade social e de manejo ambiental na Lagoa Mãe-Bá. Por sua vez, o SEBRAE-ES seria o responsável pela capacitação profissional dos artesãos, realizando cursos de design, marketing e gestão de negócios. Cerca de 20 indivíduos das 5 comunidades foram treinados para desenvolver o trabalho manual de tecelagem com base na Taboa, planta aquática abundante na região, e que tradicionalmente já era usada pela população local. Tais novos artesãos exerceriam ainda um efeito multiplicador, envolvendo dezenas de pessoas das 5 comunidades em diversas etapas da confecção de bolsas, almofadas, carteiras, chapéus, cestas, bordados, entre outros produtos.

O objetivo específico desse artigo é então compreender como as parcerias e reconhecimentos da produção artesanal do NABOA podem ser entendidos como formas de renovação territorial em curso na cidade de Anchieta (ES). De um modo mais geral, colocamos também como nossa tarefa estudar os limites dessa incomum associação entre artesanato e indústria cultural como possível mecanismo de empoderamento local e de alterações territoriais. 
Como fontes de dados para esse trabalho, usamos registros variados dos novos fluxos dos produtos artesanais. Documentos do NABOA, da SAMARCO, do SEBRAE-ES e do MERCOSUL (que reconheceu e premiou o projeto) são trazidos como parte do levantamento de dados. Em soma, recuperamos alguns registros dos próprios indivíduos que fazem parte do projeto e suas expectativas sobre os desdobramentos do projeto e a melhoria da qualidade de vida. Ao fazê-lo, esperamos conferir clareza aos processos de territorialização (física e simbólica) da região, assim como problematizar as oposições binárias entre economia e cultura, entre global e local, entre moderno e tradicional.

\section{MÉTODO}

Do ponto de vista metodológico, três desafios se mostram importantes para o presente trabalho: primeiro, definir as bases conceituais pelas quais a categoria de indústria cultural será usada; segundo, sugerir que o artesanato não é via de regra incompatível com os valores e práticas capitalistas; terceiro, propor o uso da noção de territorialização como um indicador da existência e da importância de uma certa indústria cultural vinculada ao artesanato. Tais desafios se justificam pela tentativa de oferecer um novo conjunto de elementos de investigação para o estudo do artesanato, para além da discussão da autenticidade, da tradição e da vitimização.

Nesse sentido, o primeiro passo a ser cumprido é diferenciar os sentidos de uma indústria cultural que foi definida no contexto da obra de Adorno e Horckheimer daquela que tem sido desenvolvida na Geografia Cultural. É possível afirmar que o período de emergência do debate das indústrias culturais (ascensão nazista, fordismo, massificação cultural, fascismo, alienação) por intermédio dos autores da Escola de Frankfurt influenciava fortemente o sentido pessimista dessa categoria. De forma sintética, a indústria cultural pode ser entendida como um estágio avançado do capitalismo, uma ideologia que, em adição à padronização técnica e ao controle do trabalho, estabelece uma aliança entre agentes econômicos e Estado para "produzir" um perfil médio ao cidadão. Por intermédio da cultura e de suas instituições, sobretudo naquilo que se refere aos meios de comunicação, seria possível conferir previsibilidade as ideias e aos valores no contexto de uma sociedade. De acordo com Adorno e Horckheimer, isso permitiria a pacificação dos movimentos de contestação, a subordinação da política e o aumento do consumo.

Diversas críticas foram direcionadas ao pensamento das indústrias culturais de Adorno e de Horckheimer. Em primeiro lugar, a diminuição prevista na política e no próprio indivíduo foi interpretada frequentemente como um exagero por parte dos autores, uma vez que, em última análise, representaria o "fim da história". Segundo, as cadeias produtivas de cultura não seguiram, ao longo do século XX, uma tendência absoluta de verticalização, contando com a democratização do Estado e a terceirização de serviços como mecanismos de diversificação de produtos e dos seus sentidos. Terceiro, as representações culturais tradicionais permaneceram existindo mesmo com a expansão das técnicas industriais para quase todos os países do mundo. Entre outras críticas estabelecidas, os produtos culturais capitalistas não se apresentaram completamente concentrados e com um único padrão como forma de controle social e de diminuição de custos. Ao contrário, os produtos culturais capitalistas procurariam reconhecer práticas e valores variados nas diferentes sociedades como estratégia para agregar valor. Por último, naquilo que se refere diretamente à Geografia, a indústria cultural de Adorno diminuiria o seu sentido espacial, ao afirmar que um mesmo conjunto de forças e valores seria válido para todas as partes do mundo, sem maior diferenciação.

A discussão das indústrias culturais tem se desenvolvido mais recentemente nas ciências sociais como ponderação dos limites deste modelo. Na geografia, autores como Power, Scott, Gibson, Pratt sugerem que os estágios mais recentes do capitalismo exigem diferenciação de produtos para atender a cortes distintos no mercado consumidor. Uma forma de qualificar e valorar o produto final é justamente pela atribuição de novo sentido ao trabalho artesanal, à responsabilidade social ou à sustentabilidade. Trocam-se, nesses casos, o volume e a velocidade da produção pelo valor único e original do trabalho. Tal troca indica que a cadeia produtiva pode ser conduzida por grupos econômicos com menor capital acumulado e com uma estrutura menos vertical e com menor grau de hierarquização. É nesse sentido que Scott e Power argumentam que:

[they] are growing rapidly; they tend (though not always) to be environmentally friendly; and they frequently (though again not always) employ (...) creative workers. Cultural-products industries also generate positive externalities in so far as they contribute to the quality of life in the places where they congregate and enhance the image and prestige of the local area (SCOTT; POWER, 2004, p.8). 
De fato, as parcerias que viabilizam essas indústrias culturais parecem ser constituídas por arranjos diferenciados daquele modelo de Adorno e Horckheimer. Por exemplo, nota-se que agentes públicos de diferentes níveis abrem editais de financiamento de projetos culturais, ao mesmo tempo em que ONGs e entidades internacionais estabelecem premiações, capacitações e difusões dos produtos culturais. A iniciativa privada também financia projetos ou subcontrata empresas de diferentes tamanhos para participar de tais arranjos. Podemos então dizer que a indústria cultural depende da configuração de uma estratégia comum realizada por múltiplos agentes sociais para o desenvolvimento de atividades que podem ter efeito multiplicador sobre o território. Com isso:

a number of low and middle-income countries are finding that they too are able to participate in various ways in the new cultural economy, sometimes on the basis of traditional industries and cultures. (...) an accelerating convergence between the economic and the cultural is currently occurring in modern life, and is bringing in its train new kinds of urban and regional outcomes and opening up new opportunities for policy-makers to raise local levels of income, employment and social well-being (SCOTT; POWER, 2004, p.10).

Essas novas indústrias culturais se mostram objetos legítimos para o desenvolvimento de uma investigação geográfica na medida em que parte importante do seu novo processo de valoração (material e simbólica) está ligada à territorialização. Dito de outra forma, espera-se que tais indústrias culturais busquem elementos simbólicos da vida local, assim como parte dos materiais, técnicas e trabalhadores envolvidos. Espera-se igualmente que uma parte do capital acumulado se reflita em novos equipamentos, serviços e nova qualidade de vida para a população local, ao contrário daquelas cadeias produtivas que se constroem a despeito dos interesses locais. No presente trabalho, nos escoramos na seguinte definição de territorialização:

Enquanto continuum dentro de um processo de dominação e/ou apropriação, o território e a territorialização devem ser trabalhados na multiplicidade de suas manifestações - que é também e, sobretudo, multiplicidade de poderes, neles incorporados através dos múltiplos sujeitos envolvidos (tanto no sentido de quem sujeita quanto de quem é sujeitado, tanto no sentido das lutas hegemônicas quanto das lutas de resistência - pois poder sem resistência, por mínima que seja, não existe). Assim, devemos primeiramente distinguir os territórios de acordo com aqueles que os constroem, sejam eles indivíduos, grupos sociais/culturais, o Estado, empresas, instituições como a Igreja etc. Os objetivos do controle social através de sua territorialização variam conforme a sociedade ou cultura, o grupo e, muitas vezes, com o próprio indivíduo (HAESBAERT, 2007, p. 22).

Por último, tais concepções de indústrias culturais e de terrritorialização parecem ser adequadas ao caso na medida em que os agentes locais e os diversos níveis do poder público passam a atribuir novos sentidos ao trabalho artesanal conectado ao mercado. Pelos termos do Programa do Artesanato Brasileiro (Ministério do Desenvolvimento, da Indústria e do Comércio), não se trata aqui exatamente da criação de um apoio institucional ao processo de patrimonialização do artesanato. $\mathrm{O}$ que se propõe é que tais saberes possam ser formalizados, acrescidos de novas técnicas e sentidos para que participem de forma mais ativa nos fluxos econômicos. Espera-se que os saberes artesanais se constituam em verdadeiras estratégias de inserção local na economia globalizada:

O artesanato é uma das mais ricas formas de expressão da cultura e do poder criativo de um povo. Na maioria das vezes, é a representação da história de sua comunidade e a reafirmação da sua auto-estima. Nos últimos tempos, tem-se agregado a esse caráter cultural o viés econômico, com impacto crescente na inclusão social, geração de trabalho e renda e potencialização de vocações regionais. (...) a formação de uma mentalidade empreendedora e a capacitação de artesãos para o mercado competitivo, promovendo a profissionalização e a comercialização dos produtos artesanais brasileiros (MDIC, 2012, p.7-11). 
O artesanato então se configuraria nos termos de uma indústria cultural ao realizar uma:

tentativa de formalização da diversidade por intermédio da gestão do espaço. Procuram-se então os laços tradicionais desprezados pelo ritmo da vida moderna e procura-se de algum modo encontrar uma nova posição para as suas práticas. Seja através da mobilização cívica, seja por intermédio de um reconhecimento do valor subjetivo da representação cultural via política patrimonial, ou ainda pela descoberta do potencial turístico e/ou comercial dessa prática cultural, toma-se o registro, a conservação e a reprodução dessa atividade como parte importante de uma política de diversidade. Mais do que uma pretensa tentativa de repetição do passado, se coloca que o manuseio dos símbolos e dos lugares dessa cultura permitirá em alguma medida as transformações nas relações socioespaciais de um segmento da sociedade. Nesse processo, tradição e modernidade parecem encontrar eixos de aproximação, pois as práticas artesanais, os valores antigos e as representações do passado podem ganhar novas áreas de influência. Os conhecimentos que haviam sido excluídos e substituídos por outros mais mecânicos e precisos podem então ser integrados ao mundo atual. Significa dizer que essa indústria cultural não pressupõe que a inovação se constitua pela via tecnológica, mas sim pelo know-how e pelo fortalecimento do vínculo entre produtor e consumidor (VALVERDE, 2015, p. 412).

\section{DISCUSSÃO}

A conversão da atividade artesanal das comunidades de Anchieta em uma indústria cultural exigiu uma redefinição dos agentes sociais e dos papéis que lhes são atribuídos. Foi preciso que tal atividade conduzida em Anchieta, que era realizada individualmente pelos artesãos para cumprir um papel de geração de verbas complementares, passasse a ter outros agentes participando do seu financiamento, design e comercialização. Ao criar o NABOA por intermédio de um projeto de responsabilidade social da SAMARCO, a atividade artesanal deixava de lado uma parte de suas características originais, vinculadas à subjetividade $\mathrm{e}$ irregularidade das técnicas empregadas.

As interações e as discussões entre os artesãos e os agentes da empresa geravam mudanças nos materiais empregados e nas formas de trabalho, selecionando aqueles que melhor respondiam ao projeto (comprovar o envolvimento da empresa com a solução de problemas sociais e ambientais em escala local). Apesar do trabalho realizado com a Taboa já fosse conhecido, foi preciso estabelecer, por intermédio de capacitações técnicas, as formas regulares pelas quais os artesãos passariam a exercer as suas atividades. Dessa forma, o papel do SEBRAE seria o de conferir previsibilidade à escala de produção, ao manejo da planta e à qualidade dos produtos. Como colocou Lopes:

Um grupo de 24 artesãos de 5 comunidades do entorno da Lagoa (Chapada do À, Condados, Meaípe, Porto Grande e Mãe-bá) é capacitado na técnica de retirada da vegetação (realizada de forma manual), preparo e secagem da palha/fibra, técnicas de tranças para confecção de diferentes tipos artesanatos (LOPES, 2012, p.14).

O local de desenvolvimento e de comercialização dessa atividade artesanal também começa a mudar, com a criação de uma sede para a associação, a participação em eventos e concursos nacionais e internacionais e a ampliação do mercado consumidor para muito além dos limites da cidade. Se anteriormente a prática artesanal tradicional definia uma relação direta entre o produtor e o consumidor, com uma passagem rápida de um ambiente produtivo privado para um tipo de espaço público marcado pela proximidade e pela familiaridade dos seus participantes, a nova indústria cultural estabelece um ambiente de trabalho (a sede) e define que a passagem da atividade produtiva para o circuito de consumo seja ampliada pela diversificação dos circuitos comerciais e, necessariamente, dos consumidores. De acordo com Lopes, a SAMARCO:

tem um diálogo estruturado e permanente com as comunidades vizinhas às suas unidades, fomentando o desenvolvimento local. (...) surgem projetos socioambientais de interesse das comunidades, fortalecendo, assim, a prática cidadã. (...) Uma das ações da Samarco foi a construção de um espaço de integração entre 
ela e a comunidade, o Centro de Convivência e Educação Ambiental (CCEAM). Localizado na comunidade de Recanto do Sol (...), o espaço já se tornou um ponto de encontro entre a comunidade, a Samarco, as prefeituras e demais parceiros (LOPES, 2012, p.10).

A cadeia produtiva da indústria cultural artesanal também redefine o sentido do trabalho com a Taboa. De um uso eventual realizado anteriormente, realizado pela abundância da planta e pelo reconhecimento empírico de algumas de suas características, o manejo da Taboa passa a ser justificado e conduzido por um sentido ambiental, nos limites do pensamento moderno. Por recomendações e seleções da SAMARCO e do SEBRAE, a capacitação para o trabalho com a Taboa reflete uma tentativa de controlar a distribuição da planta e de diversificar as espécies presentes nas margens da Lagoa Mãe-Bá. Assim, a prática do artesanato passa a ser qualificada por valores e selos ambientais, uma vez que a realização de certas etapas de sua cadeia produtiva permitiria uma atividade de reflorestamento e de reequilíbrio do ecossistema. Como nos apresenta Lopes:

O Programa conta com uma série de atividades, sendo as mais importantes o reflorestamento nas margens; a educação ambiental nas escolas; as oficinas de artesanato para geração de renda e os programas de estímulo à pesca de maneira sustentável (LOPES, 2012, p.15).

O próprio SEBRAE reforça essa compreensão ao afirmar que: "A atividade ajuda a controlar a mata nativa por meio de um manejo consciente. Na sede da associação trabalham 12 pessoas. As folhas da taboa secam durante 20 dias e só então os artesãos retiram a casca e começam a trabalhar" (AGÊNCIA SEBRAE, 2013). Isso significa que o processo produtivo da indústria cultural do artesanato de Anchieta depende de um sentido artesanal para alcançar uma nova valoração.

Nesse sentido, é possível classificar a atividade artesanal do NABOA como uma indústria cultural na medida em que se consolida uma estratégia para o desenvolvimento local que envolve a transposição de símbolos e práticas tradicionais para dentro de um novo sistema técnico, que gera produtos com valor agregado e traz efeitos para os artesãos e para o território. A forma pela qual se estabelece a produção e a legitimação do NABOA depende igualmente de valores que são internos e externos: os princípios ambientais que norteiam hoje sua atividade produtiva não são frutos da tradição, assim como o consumo dos produtos ultrapassou os limites das relações comunitárias.

\section{RESULTADOS}

De um conhecimento sem expressão econômica, sem coordenação e capacitação de artesãos e sem produção em escala significativa, o artesanato em Anchieta (ES) passa por um processo de profissionalização. De acordo com Karoline: "O NABOA já confeccionou mais de 15 mil peças artesanais, em torno de 200 variedades de produtos, que são vendidos na sede do Núcleo, em Mãe-Bá - Anchieta, além das lojas de revenda em todo o Brasil e no exterior" (KAROLINE, 2013).

Atualmente, cerca de 20 artesãos e 130 colaboradores garantem que milhares de peças estejam a disposição não apenas dos visitantes da cidade de Anchieta, mas também alcancem países como França, Israel e Itália, entre outros destinos. A maior regularidade no conhecimento, a formação de novos artesãos e as parcerias com ONGs, instituições públicas e agentes privados garantem que esses fluxos tomem sentido econômico e também assumam elementos de nova territorialização.

Se o trabalho com a Taboa já fazia parte dos conhecimentos das comunidades locais, a conexão com a SAMARCO e com o SEBRAE-ES alterou o sentido e a escala da produção. Há em curso um processo de uniformização e regularização técnica dos produtos. As formas e funções desses produtos também não são mais criadas necessariamente para servir aos fins da vida social que se desenrola na escala local: tais produtos passam a ser criados como parte de uma cadeia de comercialização que alcança o exterior, com valores que são consumidos e vivenciados em outras escalas. Nesse contexto, a autenticidade (no sentido patrimonial do termo) da produção artesanal de Anchieta não pode mais ser facilmente defendida, pois parte importante do processo criativo e de usufruto desses produtos passam a ser realizados por outros. De acordo com Karoline: 
Artesãos das comunidades de Mãe-Bá, Porto Grande, Condados e Chapada do Á, todas localizadas no entorno da Lagoa de Mãe-Bá, na divisa dos municípios de Guarapari e Anchieta, formam a associação [NABOA]. Desde a fundação, o grupo recebeu apoio do Sebrae no Espírito Santo para atuação no mercado de trabalho. Por meio de capacitação e consultoria de design, a instituição desenvolveu várias coleções e projetos que foram bem aceitos no mercado (KAROLINE, 2013).

Constrói-se uma duplicidade entre o empoderamento dos artesãos e o rompimento parcial dos seus laços tradicionais, consolidando uma indústria cultural por intermédio da redefinição das relações entre produtores, conhecimento e consumidores. Tal duplicidade permite mudanças na condição material de vida e modernização dos costumes. Ainda assim, o que se vende como valor é o "resgate da tradição", tal qual Alexandre Passos, Secretário de Turismo (ES), exprime:

$\mathrm{O}$ artesanato é parte importante dos atrativos de uma cidade e tem na atividade turística sua maior aliada. É crescente o interesse dos turistas por produtos artesanais, como forma de (...) resgate da cultura local (PASSOS Apud. SECRETARIA DE TURISMO - ESTADO DO ESPÍRITO SANTO, 2013).

Cada nova premiação concedida em escala regional, nacional ou internacional significa uma ampliação da rede de circulação e de parcerias que marca as atividades do NABOA. Aos prêmios específicos às atividades artesanais (MERCOSUL, UNESCO), somam-se as premiações referentes à responsabilidade social e à sustentabilidade (Prêmio Brasil Ambiental/AMCHAM-Rio). Os produtos passam então a ter a sua confecção orientada para cumprir o rigor dos editais e o interesse de consumidores internacionais. De acordo com a Agência Sebrae:

Com o incentivo do Sebrae ao artesanato capixaba, a associação já foi premiada 14 vezes. Um deles veio da Unesco, devido ao trabalho de preservação ambiental, sustentabilidade e geração de renda realizado pelo grupo do Espírito Santo. Em outro prêmio concedido pelo Mercosul, a comunidade ficou em primeiro lugar entre 96 concorrentes, graças a uma fina bolsa feita com taboa, que virou referência do artesanato capixaba. Os artesãos produzem quase 300 itens e faturamento médio chega a R\$ 12 mil por mês (AGÊNCIA SEBRAE, 2013).

Tal processo fica claro com o exemplo de uma nova capacitação técnica (Comunidade Criativa) criada para os artesãos do NABOA, que é voltado à adaptação de seus conhecimentos e produtos para uso nos circuitos nacional e internacional da indústria da moda e do design. Dessa forma, espera-se atribuir novo valor e novo alcance aos produtos artesanais:

O curso [Comunidade Criativa] visa capacitar as artesãs com técnica e/ou produto dirigido ao segmento de moda, com design inspirado em referênciasbrasileiras. Objetivou, também, valorizar as capacidades locais, incentivar o comércio justo e promover a inclusão destes grupos na indústria da moda. A matéria-prima usada foram as conchas e escamas recolhidas nas praias de Anchieta. A capacitação do curso foi coordenada por Heloísa Crocco, que é um dos principais nomes da junção design e artesanato no país (SIMNOTICIAS, 2013).

Com isso, vê-se a modernização das práticas culturais como parte importante do desenvolvimento territorial, contemplando novos equipamentos (sede do NABOA), novos conhecimentos, novos fluxos de capital e novo poder de pressão sobre o poder público para a melhoria da infraestrutura na área de entorno. Também cabe ressaltar o ganho em autoestima que define um ciclo de relações positivas entre os moradores das comunidades e a área a sua volta. De acordo com Cesar Weslley Conti Nunes, presidente do NABOA: 
A participação da Samarco Mineração foi de vital importância para atingirmos nossos objetivos e a parceria com o SEBRAE foi e continua sendo de relevância, porque além da qualificação profissional, deu e dá credibilidade ao projeto. Também destacamos a excelência da gestão que conseguimos implantar na Associação a ponto de sermos atualmente, a única Associação de Artesanato no Estado que é cadastrada nos Ministérios do Turismo e Desenvolvimento Social, com CNPJ, Inscrição Estadual, Municipal que emite suas próprias Notas Fiscais. Os associados, atualmente desfrutam de qualidade de vida bem melhor do que tinham antes, mas são conscientes de que alcançar o topo foi árduo e trabalhoso! Alguns até retornaram aos bancos escolares depois de vários anos afastados deles (César Weslley Conti Nunes, Apud. ARRUDA, 2009).

\section{CONCLUSÕES}

É possível afirmar que o grande desafio do desenvolvimento de uma indústria cultural ligada ao artesanato tal qual se observa em Anchieta (ES) é justamente aquele de garantir a estabilidade do desenvolvimento territorial e aumentar o caráter criativo originado dos valores dos próprios artesãos.

É preciso reconhecer que tais ganhos territoriais observados pelos agentes do NABOA e seus parceiros são em parte dependentes da regularidade da demanda externa à área. Uma alteração nos circuitos de comercialização pode romper o ciclo de desenvolvimento territorial experimentado. De modo adicional, uma alteração na política de reconhecimento ou de financiamento da cultura, seja em escala nacional, seja em escala internacional, pode ter graves consequências na estabilidade dos fluxos que tem mantido o ciclo atual de empoderamento e de desenvolvimento territorial.

No contexto da paralização das atividades da Complexo de Usinas de Pelotização da Samarco em Anchieta, já em 2016, devido às multas e suspensões de licenças ambientais, políticas de responsabilidade social e sustentabilidade se mostram matéria ainda mais sensível (VIGNOLI, 2016). A paralização afeta parte decisiva do orçamento da cidade, pressionando cortes nos gastos e ao mesmo tempo sinaliza uma possibilidade de reconstrução de uma política de legitimização das ações. Para superar o julgamento negativo na opinião pública devido às consequências da atividade produtiva, faz-se uso de produções de objetos culturais que comportam algum efeito territorial na microescala.

É preciso ainda considerar que a dependência das capacitações relativiza o caráter criativo local e diminui a representatividade dos símbolos e valores locais que se mostram presentes nos produtos finais. Isso pode acarretar em uma tendência ao descolamento afetivo e político da vida social local em relação à atividade produtiva. Nesse processo, o valor social e cultural ligado ao território poderia diminuir em médio prazo, ainda que sua base material apresente ganhos.

Por último, é preciso igualmente evitar que o NABOA seja totalmente apropriado como parte de uma estratégia de legitimação por parte de grandes agentes econômicos, conferindo assim menor sentido social as suas ações. O interesse da SAMARCO na confirmação de sua responsabilidade social e ambiental não pode ser confundido diretamente com os limites das práticas do NABOA, sob risco de instabilidade na indústria cultural do artesanato de Anchieta.

\section{REFERÊNCIAS}

ADORNO, T.W; HORCKEIHEIMER, M. Indústria cultural e sociedade. São Paulo: Paz e Terra, 2002.119p.

AGÊNCIA SEBRAE. Núcleo capixaba que faz arte com taboa é referência mundial Planta aquática é usada como matéria-prima para fabricar bolsas, caixas e até móveis. Disponível em: < http://www.agenciasebrae.com.br/noticia/21088527/ultimasnoticias/nucleo-capixaba-que-faz-arte-comtaboa-e-referencia-mundial/ >. Acessado em: 12 de Set de 2013.

ARRUDA, V. Arte se faz NABOA com Taboa. Revista Plus, nº 4, 2009. p.8-9.

GIBSON, C. Cultures at work: why 'culture' matters in research on the 'cultural' industries. Social \& Cultural Geography, Volume 4, Issue 2, p. 201-215, 2003.

HAESBAERT, R. Território e Multiterritorialidade: Um Debate. GEOgraphia, Ano IX, nº 17, p. 19-46, 2007. 
KAROLINE, A. Artesãos apoiados pelo Sebrae ganham prêmio da Unesco - O grupo NABOA concorreu com outros países do Mercosul. Disponível em: < http://www.agenciasebrae.com.br/noticia/18760412/ultimasnoticias/artesaos-apoiadospelo-seb rae-ganham-premio-da-unesco/ >. Acessado em: 12 de Set de 2013.

LOPES, C.M.A. Taboa Lagoa: um Caso de Inovação e Desenvolvimento Sustentável da Samarco Mineração S.A. Anais do IX Simpósio de Excelência em Gestão e Tecnologia, 2012.

Ministério do Desenvolvimento, Indústria e Comércio Exterior (MDIC). Base conceitual do artesanato brasileiro. Brasília: MDIC, 2012. 63p.

MITCHELL, D. Não existe aquilo que chamamos de cultura. Espaço e Cultura, p. 81-101, 2008.

POWER, D.; SCOTT, A.J. Cultural industries and the production of culture. London: Routledge, 2004. $384 \mathrm{p}$.

PRATT, A.C. The Cultural Economy: A Call for Spatialized 'Production of Culture' International Journal of Cultural Studies, vol. 7, no 1, p. 117-128, 2004.

SECRETARIA DE TURISMO DO ESTADO DO ESPÍRITO SANTO. Artesanato Capixaba: prêmio na Unesco pode impulsionar o turismo em Anchieta. Disponível em: <http://www.turismo.es.gov.br/index.php?id=/comunicacao/noticias/materia.php\&cd_matia=1846>. Acessado em: 10 de Set de 2013.

SIMNOTICIAS. Artesanato de Anchieta e região será usado em desfile em São Paulo. Disponível em: < http://www.simnoticias.com.br/2012/index.php/noticias-2/item/676-artesana to-de-anchieta-eregi\%C3\%A3o-ser\%C3\%A1-usado-em-desfile-ems\%C3\%A3o-paulo >. Acessado em 15 de Set de 2013.

VALVERDE, R.R.H.F. A Indústria Cultural como objeto de Pesquisa Geográfica. Revista do Departamento de Geografia da USP, v.29, P. 391-418, 2015.

VIGNOLI, E. Samarco parada faz Anchieta deixar de arrecadar R\$ 9 milhões no Espírito Santo. Disponível em: < http://g1.globo.com/espirito-santo/desastre-ambiental-no-rio-doce/noticia/2016/03/samarco-paradafaz-anchieta-deixar-de-arrecadar-r-9-milhoes-no-es.html >. Acessado em: 01 de Jun de 2016. 